\title{
Development and application of a transdermal device derived from carboxymethyl cellulose and acid functionalized carbon nanotube
}

\author{
Barun Mandal and Sagar Pal* \\ Polymer Chemistry Laboratory, Department of Applied Chemistry, Indian School of Mines, \\ Dhanbad-826004, Jharkhand, India. \\ *Email: barunmandaliitd@gmail.com
}

To overcome the problems associated with conventional polymer hydrogel in transdermal drug delivery like limited penetration ability and poor stability, herein, for the first we report a new generation of hydrogel nanocomposite based on carboxy functionalised multiwalled carbon nanotube and carboxymethyl cellulose [1-3].

The developed nanocomposite hydrogel is well characterized and found to be biocompatible (Figure 1 and 2).

Finally the composite demonstrates excellent potential towards sustained release of transdermal drug - diclofenac sodium (Figure 3).

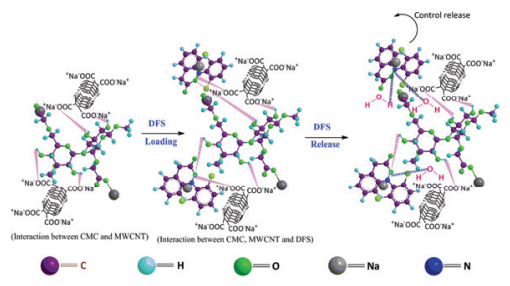

Graphical abstract
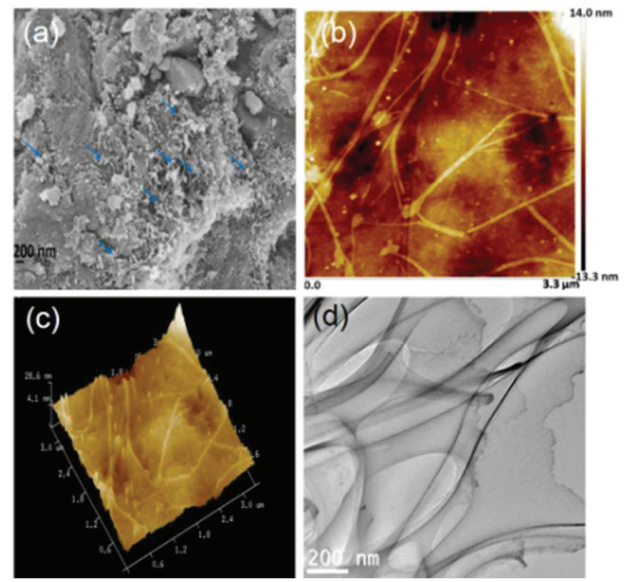

Figure 1: (a) FESEM, (b) 2D topographic, (c) 3-D topographic and (d) TEM images of CMC-MWCNT nanocomposite
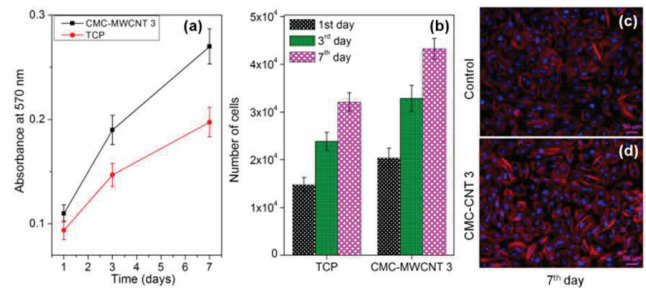

Figure 2: (a) Cell viability result, (b) cell proliferation result of control and CMC-MWCNT nanocomposite study by MTT assay ( $\mathrm{SD} \pm 3$ ), and (c), (d) cellular attachment of RFBs by rhodaminephalloidin and DAPI assay at different time periods

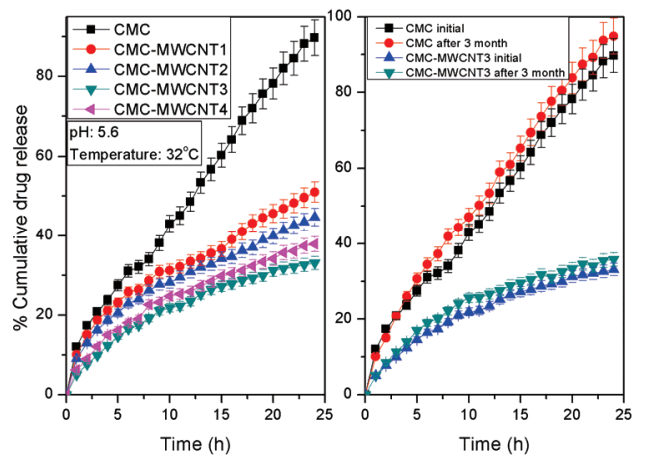

Figure 3: Diclofenac release from (a) CMC and various nanocomposites and (b) stability study of drug loaded CMC and best grade of CMC-MWCNT nanocomposite for 3 months

\section{References}

1. Barun Mandal, Dipankar Das, Arun Prabhu Rameshbabu, Santanu Dhara and Sagar Pal*. RSC Advances, 2016, 6, 19605.

2. J. R. Walter, S. Xu, Drug Discovery Today, 2015, 20, 1293-1299.

3. R. Sridhar, R. Lakshminarayanan, K. Madhaiyan, V. A. Barathi, K. H. C. Limh,S. Ramakrishna, Chem. Soc. Rev., 2015, 44, 790-814. 